\title{
Strategies for the management and prevention of conformation-related respiratory disorders in brachycephalic dogs
}

This article was published in the following Dove Press journal:

Veterinary Medicine: Research and Reports

4 June 2015

Number of times this article has been viewed

\author{
Rowena MA Packer' \\ Michael S Tivers ${ }^{2}$ \\ 'Department of Clinical Science \\ and Services, Royal Veterinary \\ College, University of London, \\ London, ${ }^{2}$ School of Veterinary \\ Sciences, University of Bristol, \\ Bristol, UK
}

Correspondence: Rowena MA Packer Department of Clinical Science and Services, Royal Veterinary College, University of London, Hawkshead Lane, London AL9 7TA, UK Tel +44 I707 66606 I

Email rpacker@rvc.ac.uk
Abstract: Brachycephalic (short-muzzled) dogs are increasingly popular pets worldwide, with marked increases in registrations of breeds such as the Pug and French Bulldog over the past decade in the UK. Despite their popularity, many brachycephalic breeds are affected by an early-onset, lifelong respiratory disorder, brachycephalic obstructive airway syndrome (BOAS). This disorder arises due to a mismatch in the proportions of the skull and the soft tissues held within the nose and pharynx, resulting in obstruction of the airway during respiration. Increased airway resistance encourages secondary changes such as eversion of the laryngeal saccules and collapse of the larynx. Clinical signs of BOAS are often early onset and chronic, including dyspnea, exercise intolerance, heat intolerance, and abnormal and increased respiratory noise. Episodes of severe dyspnea can also occur, leading to cyanosis, syncope, and death. BOAS may have a severe impact upon the welfare of affected dogs, compromising their ability to exercise, play, eat, and sleep. Although a well-described condition, with surgical treatments for the palliation of this disorder published since the 1920s, many dogs still experience airway restrictions postsurgically and a compromised quality of life. In addition, the prevalence of this disorder does not appear to have substantially reduced in this time, and may have increased. Ultimately, strategies to improve the breeding of these dogs to prevent BOAS are required to improve brachycephalic health and welfare. Recent studies have revealed conformational risk factors associated with BOAS, such as short muzzles and thick necks, which should be discouraged to avoid perpetuating this serious disorder. Positive changes to brachycephalic health may be impeded by a perception of BOAS being "normal for the breed". This perception must be avoided by owners, breeders, and vets alike to prevent undertreatment of individuals and the perpetuation of this serious disorder to future generations of dogs.

Keywords: short-muzzled, canine welfare, airway obstruction, dyspnea

\section{Brachycephalic dog breeds and their physiology}

Short-muzzled dogs are becoming increasingly popular pets worldwide. For example, the Pug has experienced a greater than $730 \%$ increase in annual registrations with the Kennel Club (UK) between 2002 and 2013 (rising from 1,105 registrations per year to 8,071 ) and the French Bulldog a greater than 2,708\% increase (rising from just 247 to 6,690 new dogs per year). ${ }^{1}$ These, along with other characteristically shortmuzzled breeds such as the Bulldog, Shih Tzu, Boston Terrier, and Pekingese are termed brachycephalic (from the Latin roots for "short" and "head"). Brachycephaly is a discrete skeletal mutation, ${ }^{2}$ where early ankylosis in the basicranial epiphyseal cartilage leads to altered growth of the basioccipital and basisphenoid bones, manifesting as a shortening of the basicranial axis. ${ }^{3}$ In these breeds, while the lower jaw 
is of a relatively normal length, the upper jaw is markedly foreshortened, resulting in a characteristically short muzzle. Several metrics have been used to define and describe the degree of brachycephaly, including the craniofacial ratio: the muzzle length to the cranial length, ${ }^{4}$ the skull width-to-length ratio, ${ }^{5}$ the cranial length to skull length, ${ }^{6}$ and the craniofacial angle. ${ }^{7}$ Due to variation in definitions, there is no definitive list of brachycephalic breeds, and indeed, variation within some breeds may mean that the definition is more suited for the individual dog rather than the breed as a whole.

\section{Breeding brachycephalic dogs}

For several brachycephalic breeds, this phenotype was originally positively selected for, as a potential advantage in fighting ${ }^{8}$ with their craniofacial conformation enabling increased biting forces. ${ }^{9}$ In contrast, this feature was selected for in other brachycephalic breeds for their appeal as "lap dogs" or for a companion role. ${ }^{10}$ It is thought that the childlike features of brachycephalic dogs, such as large, round, wide-set eyes and rounded faces, are instinctively attractive to humans. ${ }^{11}$ The brachycephalic phenotype is described in the various breed standards, which are the "blueprint" describing the desirable features of each breed. The terminology referring to brachycephaly often refers to the relative shortness of the "muzzle", or "face". This is described in the Pug as "Muzzle relatively short, blunt, square", ${ }^{12}$ in the Bulldog as "Face relatively short", ${ }^{13}$ and in the Pekingese as "Muzzle must be evident, but may be relatively short and wide". ${ }^{14}$ These conformations have been taken to extremes in some individuals, where exaggerated brachycephalic morphologies exhibit little evidence of a muzzle, and in profile appear almost completely flat (Figure 1).

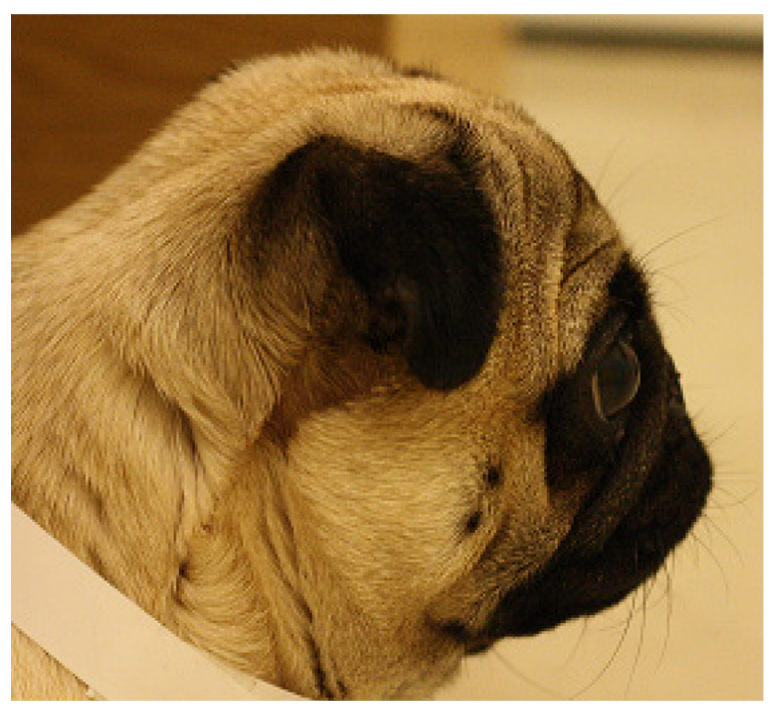

Figure I Extreme brachycephalic morphology in a Pug.

\section{Respiratory problems and airway collapse in brachycephalic dogs}

It has been stated that by selectively breeding to reduce the bony framework of an organ to "less than a third of its natural size", there are inevitable major consequences on the structures contained within it. ${ }^{15}$ Brachycephalic obstructive airway syndrome (BOAS) (also referred to as brachycephalic airway syndrome or brachycephalic airway obstructive syndrome) describes a spectrum of disease that involves partial or complete obstruction of the upper airway during respiration, leading to restricted breathing. As the name of the disorder implies, BOAS affects brachycephalic dogs. BOAS has been reported in over ten brachycephalic breeds internationally, including the Bulldog, Pug, French Bulldog, Boxer, Shih Tzu, Boston Terrier, Pekingese, Staffordshire Bull Terrier, Shar Pei, Rottweiler, Chow Chow, Pomeranian, Bullmastiff, Lhasa Apso, and Cavalier King Charles Spaniel. ${ }^{4}$ Recent research has quantitatively linked BOAS with the brachycephalic phenotype, with the risk of BOAS significantly increasing as the relative length of the muzzle decreases. ${ }^{16}$ Despite changes in phenotype over time, BOAS was recognized as a possible consequence of brachycephaly over 50 years ago, with statements from the 1960s such as "Present day trends in the breeding of brachycephalic dogs produce specimens which suffer from dyspnoea to an everincreasing degree". ${ }^{17}$

\section{Clinical signs of BOAS}

BOAS is characterized by clinical signs such as dyspnea, exercise intolerance, and abnormal and increased respiratory noise including stertor and stridor. Episodes of severe dyspnea can also occur, leading to cyanosis, hyperthermia, and syncope. ${ }^{18}$ Minor aggravations can lead to severe respiratory distress, ${ }^{19}$ with physiological arousal associated with both negative and positive experiences (eg, stress, but also exercise and excitement) acting as aggravators that can lead to respiratory crises. ${ }^{19,20}$ Severely affected individuals exhibit labored breathing, even at rest, adopting a wide stance with their elbows abducted from their chest, with the use of accessory abdominal musculature ${ }^{21}$ and over-inflation of the chest. ${ }^{20}$ BOAS-affected dogs are also prone to heat stroke, due to impaired heat exchange (see "Nasal obstruction"), which can result in death. ${ }^{19}$ BOAS has the capacity to affect the dog while both awake and asleep, with sleep-disordered breathing (including episodes of apnea) previously recorded in the Bulldog. ${ }^{22}$ Clinical signs are often severe by 12 months of age ${ }^{23}$ and are lifelong thereafter, sometimes even with medical and surgical management (Figure 2). 


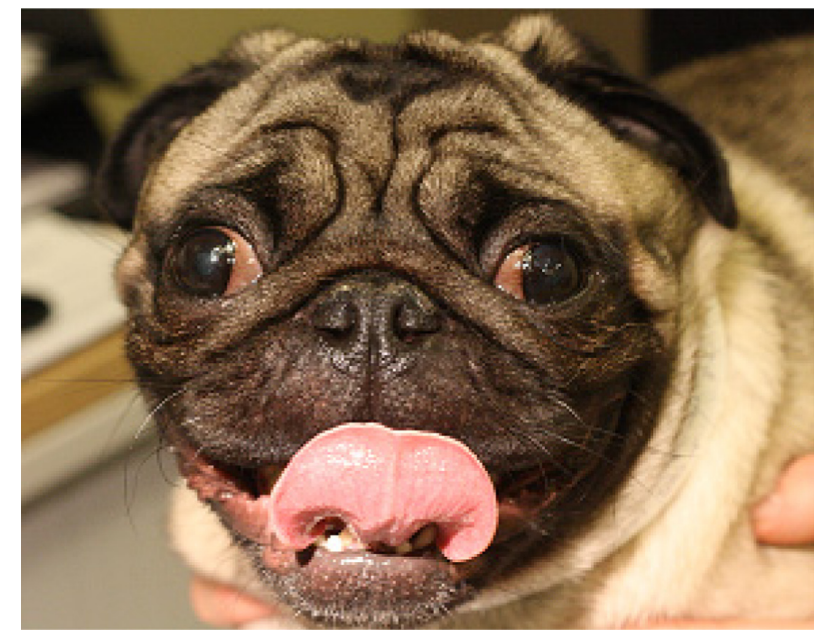

Figure 2 Pug diagnosed with brachycephalic obstructive airway syndrome exhibiting respiratory distress preoperatively.

\section{Components of BOAS and pathophysiology}

These clinical signs are caused by primary and secondary abnormalities of the upper airway leading to restricted breathing. This review focuses on these abnormalities, and the potential for surgical and medical management to reduce their impact upon the respiration and quality of life (QoL) of affected dogs.

\section{Abnormalities of the soft palate and nasopharynx}

The shortening of the skeletal muzzle that results in the brachycephalic phenotype appears not to be accompanied by corresponding soft tissue shortening, leading to a mismatch in proportion. Reduction of the oral cavity volume results in a relative oversize of several soft tissue structures within it, particularly the soft palate, the tongue, and the tonsils. This creates a cramming effect within the skull, with too much tissue in the available space. ${ }^{24}$ This "excess" soft tissue reduces the space available for airflow, partially blocking the nasopharynx and the larynx and interfering with the passage of air during inspiration and expiration. ${ }^{24}$

Soft palate elongation and thickening is the most common anatomical abnormality diagnosed in dogs with BOAS, seen in $85 \%-100 \%$ of BOAS cases. ${ }^{18,20,25-27}$ In mesocephalic breeds, the soft palate continues caudally from the hard palate, and passes just caudal to the last upper molar. ${ }^{5}$ In contrast, in brachycephalic breeds, the junction of the hard and soft palates is more caudal to this transverse level. Thus, the soft palate may partially block the larynx, interfering with the passage of air during inspiration and expiration. The overlong and over-thick soft palate can be seen on a radiographic examination or computed tomography (CT) scan, often extending $1-2 \mathrm{~cm}$ beyond the epiglottis. ${ }^{20}$ In addition, macroglossia (an overly long or thick tongue) ${ }^{28}$ redundant or hypertrophic pharyngeal folds, ${ }^{29}$ and hypertrophic tonsils ${ }^{15}$ are also reported in brachycephalic dogs, contributing to obstruction. This excess tissue in the laryngeal region has also been observed to be drawn into the rima glottidis on inspiration. ${ }^{15}$ Increased airway resistance is often manifested in altered respiratory noise, with the nature and magnitude associated with the site and severity of obstruction. In animals with minimal obstruction, slight stertor is often the only easily detectable abnormality. ${ }^{30}$ This is caused by the relatively elongated soft palate being transiently inhaled into the larynx, with the sound heard over the pharynx correlating on fluoroscopy with this event. ${ }^{22}$ Stertor has been reported in brachycephalic dogs while awake and asleep, and alongside episodes of sleep apnea (Figure 3). ${ }^{22}$

\section{Nasal obstruction}

The majority of the total airway resistance, from the nares to the bronchioles, occurs in the nasal cavity. ${ }^{31}$ Further obstruction of the airways is present at the external nares and inner nasal vestibule in many brachycephalic dogs. The wing of the nostil (ala nasi) is congenitally deformed in many brachycephalic dogs, ${ }^{32}$ with a decrease in the transverse diameter of the airway. This causes increased resistance to airflow, and results in increased inspiratory effort. ${ }^{23}$ This obstruction can be heard as a high-frequency whistling over the nares. ${ }^{22}$ The so-called stenotic nares have been reported to affect half of all BOAS cases; $;^{20,24,25}$ however, much higher levels of $80 \%-100 \%$ of cases have been reported in other studies. ${ }^{26,28,33}$ This narrowing of the external nares functionally restricts ala nasi mobility, impeding the abduction normally observed in healthy dogs to facilitate airflow (Figure 4$).{ }^{15}$

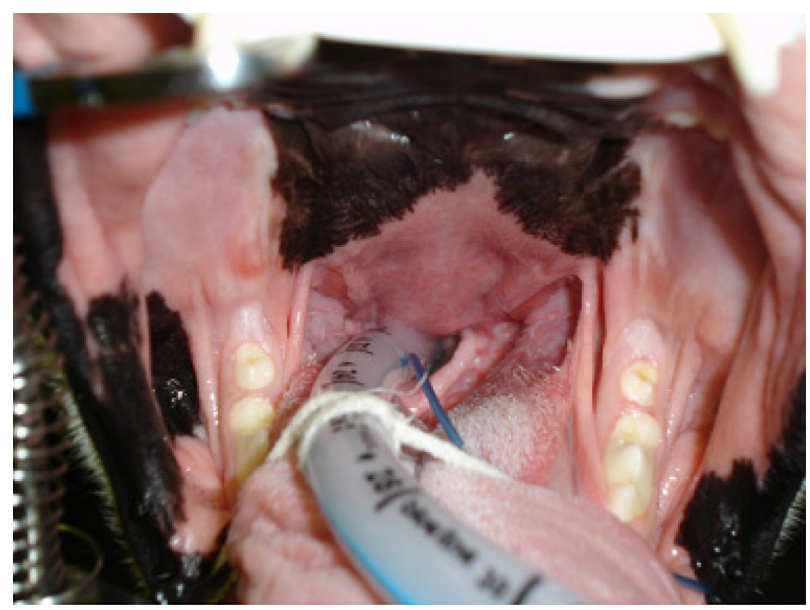

Figure 3 Elongated soft palate in a Bulldog. 

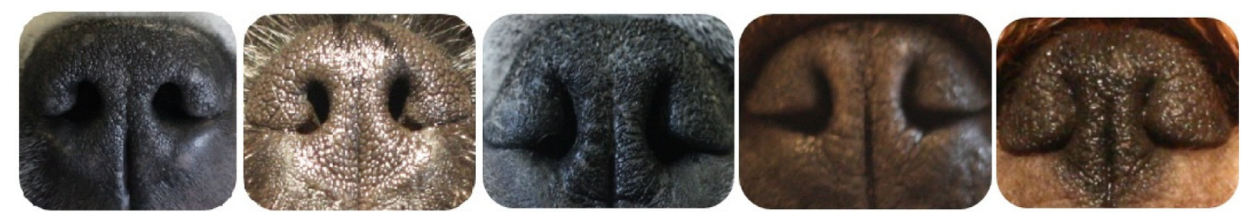

Figure 4 Stenotic nares in brachycephalic dogs, increasing in severity from left to right.

In addition to external narrowing, the inner nasal vestibule is further narrowed due to the relatively large inner wing of nasal cartilage. ${ }^{34}$ In some dogs, stenosis may be mild, but in others, it may be almost complete, with dogs forced to open-mouth breathe almost continually. ${ }^{35}$ Inhalation and exhalation through the nose are observed in resting dogs when the ambient temperature is $<26^{\circ} \mathrm{C}$, and when dogs run at slow speeds in the cold $\left(10^{\circ} \mathrm{C}\right) .{ }^{36}$ The dog's nose is specialized to dissipate heat through the nasal conchae. These are covered in a large, vascularized mucous membrane, which cools the air by evaporation during inspiration. ${ }^{37}$ Both lingual and nasal blood flow increase during panting, to facilitate heat exchange. ${ }^{38}$ The lateral nasal gland (glandula nasalis lateralis) facilitates this process of rapid heat exchange via secreting fluid into the nasal vestibule and onto the conchae, potentiating evaporation. The function of these glands is analogous to that of sweat glands in humans. ${ }^{39}$ This allows effective thermoregulation, and accounts for $19 \%-36 \%$ of the increase in respiratory evaporation associated with thermal panting. ${ }^{39}$ As such, restricted nasal ventilation has a great impact on dogs' thermoregulatory abilities.

Marked changes in the architecture within the nose of brachycephalic dogs further impact upon this evaporative process, impairing thermoregulation.$^{40}$ In the growing animal, the turbinates develop and expand, filling the nasal cavity. Crucially, expansion stops before the mucosal covering of the turbinate lamellae contact, allowing small air gaps between adjacent lamellae, thus facilitating local airflow. ${ }^{41}$ In brachycephalic dogs, turbinate growth in the young dog continues despite inhibition of growth of the midface, resulting in relatively oversized turbinates. This causes contact between turbinate lamella mucosal surfaces, impeding nasal airflow. Abnormal nasopharyngeal turbinates were diagnosed in over $20 \%$ of BOAS cases in a recent study. ${ }^{42}$ Intranasal airway resistance is further increased by abnormal growth of the conchae into the nasal meatus, ${ }^{43}$ either caudally into the nasopharyngeal meatus (caudal aberrant conchae) or rostrally into the middle and ventral meatus (rostral aberrant conchae). ${ }^{44}$ The structure of the conchae has also been noted as abnormal, with relative thickening of the lamellae for the size of the dog. ${ }^{45}$ Rhinomanometric studies measuring solely nasal airflow resistance confirmed that intranasal resistance is significantly higher in brachycephalic dogs compared to normal dogs. ${ }^{46,47}$

\section{Secondary changes}

The culmination of these obstructive abnormalities can lead to a functionally impaired dog with clinical signs that limit its physical capabilities. These primary abnormalities can result in markedly increased respiratory efforts to overcome this resistance, encouraging collapse of the airway. ${ }^{29} \mathrm{In}$ response to the increased effort to exhale, pleural pressure increases above atmospheric pressure. This in turn dramatically increases transmural pressure across the wall of the intrathoracic portion of the respiratory tract, which can then collapse..$^{33}$ This then becomes a vicious cycle, due to the compressed airway causing air to accelerate through the collapsed portion. Due to the Bernoulli effect, the increased velocity through the narrowed airway leads to a further decrease in pressure within the collapsed airways, encouraging further narrowing. ${ }^{33}$ This means that affected brachycephalic dogs must generate large pressure changes during inhalation and exhalation, which may result in secondary changes, most importantly laryngeal collapse. ${ }^{48,49}$ The pliable and soft cartilages of young animals may become fixed and collapse following continued forced exhalation. ${ }^{33}$ Changes to the airway induced by chronic inspiratory effort include eversion of the laryngeal saccules - the first stage of laryngeal collapse. ${ }^{50}$ If this is left untreated, further collapse of the laryngeal cartilages can occur, firstly medial displacement of the cuneiform processes of the arytenoid cartilages (grade II laryngeal collapse), followed by collapse of the corniculate processes of the arytenoid cartilages with loss of the dorsal arch of the rima glottis (grade III laryngeal collapse). ${ }^{51}$ Increasing severity of obstruction may result in episodes of syncope, or even death from suffocation..$^{21,52}$ Stridor, described as audible wheezing, is often associated with restricted airflow at the level of the larynx, and is a common manifestation of laryngeal collapse. ${ }^{18}$ Laryngeal collapse was recently diagnosed in brachycephalic dogs less than 6 months of age (Figure 5). ${ }^{53}$ 


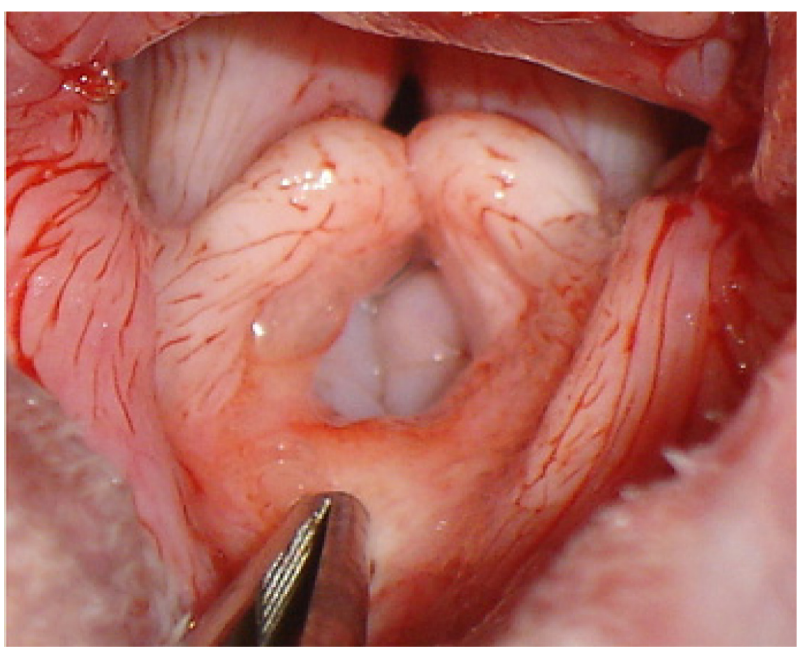

Figure 5 Close-up image of grade II laryngeal collapse.

Notes: Note the everted laryngeal saccules and medial collapse of the cuneiform processes of the larynx. This dog had undergone a folded flap palatoplasty prior to excision of the everted saccules.

\section{Co-occurring abnormalities}

Tracheal hypoplasia is a congenital condition in which the tracheal cartilages overlap or oppose to form a Q-shaped structure, ${ }^{24}$ resulting in a reduced tracheal diameter/thoracic inlet ratio $(\mathrm{TD} / \mathrm{TI}) .^{54}$ This condition is frequently seen in brachycephalic dogs, with overrepresentation in Bulldogs. ${ }^{54}$ Although not considered a component of BOAS, tracheal hypoplasia can exacerbate clinical signs in affected dogs. ${ }^{42}$

\section{Diagnosis}

Initial veterinary evaluation involves taking a history of clinical signs, along with an assessment of the degree of respiratory compromise. Clinical signs may or may not be overtly present in the consult room (this may depend upon the stress level of the dog at that time and the ambient temperature). Close questioning of the owners regarding their dog's behavior, respiratory noise, and appearance during different activities (eg, while on a walk, playing, eating, or sleeping) may reveal the true extent of their compromise. Physical examination includes laryngeal, tracheal, and thoracic auscultation to determine the location and assess the quality of respiratory noise. In addition, respiratory rate, mucous membrane color, and characteristics of respiratory noise can help determine severity. If clinical signs are not present at rest, a short exercise challenge such as the 6-minute walk test may be carried out to evaluate the dog's ability to cope with increased activity. ${ }^{55}$ This test is specifically aimed at assessing overall cardiopulmonary function; however, it measures the ability to exercise as a surrogate of this.
Stenotic nares are comparatively easy to diagnose, but the severity of stenosis for individual cases may be debatable, due to diagnosis historically being carried out via a subjective visual assessment. ${ }^{56}$ Recent studies have quantified this assessment with the development of the "nares" ratio, reflecting the degree of external stenosis. ${ }^{4}$ It is important to recognize that visual examination of the external nares does not provide information on the degree of intranasal obstruction.

Examination of the oropharynx in the conscious animal is difficult and unrewarding, and can induce extreme distress. Therefore, the diagnosis of intraoral upper airway abnormalities is generally performed under general anesthesia. ${ }^{35}$ Diagnosis under general anesthesia is typically combined with immediate surgery to relieve any obstructions present, ${ }^{57}$ as anesthesia is of high risk in brachycephalic breeds. ${ }^{18}$

Thoracic radiography is indicated for assessment of the lower airway and for any concurrent problems. Thoracic radiographs are used to diagnose tracheal hypoplasia, defined as the ratio of the tracheal lumen diameter and the thoracic inlet (TD/TI) being $<0.16 .^{21,29}$ These images can also be used to detect concurrent problems such as aspiration pneumonia, pulmonary edema, and hiatal hernia, which are associated with BOAS. In some animals, a positive-contrast esophagram under fluoroscopy may be necessary to confirm a diagnosis of hiatal hernia. Aspiration pneumonia may be related to airway impairment and regurgitation. In one study, $4.7 \%$ of dogs were affected with aspiration pneumonia preoperatively. ${ }^{27}$ Advanced diagnostic imaging such as CT or magnetic resonance imaging (MRI) can be used to assess airway abnormalities in greater detail for surgical planning, and to detect further abnormalities (eg, intranasal cysts ${ }^{58}$ ) but may not be warranted in all cases (Figure 6).

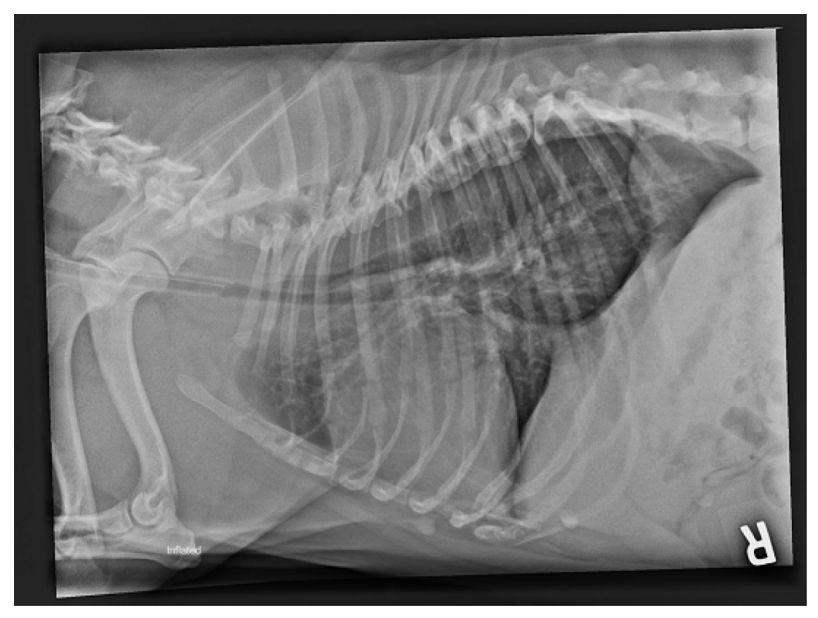

Figure 6 Lateral thoracic radiograph of a Bulldog with a hypoplastic trachea. 


\section{Surgical management, complications, and anesthesia}

Surgical management of BOAS is indicated to reduce airway obstruction and improve the dog's QoL. Surgical treatment typically involves procedures to ameliorate the degree of obstruction to the upper airway, improving clinical signs in the short term and potentially preventing or reducing further deterioration in function. However, surgery is associated with significant morbidity and mortality (see "Postoperative outcomes and QoL"), and thus, there must be a balance between potential QoL gains and risks of these procedures. ${ }^{59}$

\section{Presurgical considerations}

Sedation and premedication should be considered for dogs that are anxious and for those scheduled for BOAS surgery. These medications can avoid stresses that increase their oxygen demand that may exacerbate respiratory distress, and can increase the ease of handling preoperatively. Sedative-anxiolytic drugs such as acepromazine in conjunction with an opioid have been recommended ${ }^{60}$ Despite their advantages, drugs used during sedation and premedication can relax the smooth muscle of the naso- and oropharyngeal region, ${ }^{61}$ risking further airway obstruction, and thus, dogs should be continually observed once sedated, with the team prepared for an emergency. In addition, these drugs are also associated with decreased tear production, leading to corneal drying. ${ }^{6}$ Due to the exophthalmic, exposed corneas seen in many brachycephalic dogs, eye lubrication should be used where appropriate to avoid corneal ulceration. Following premedication, an intravenous catheter should be placed as soon as possible.

Intravenous anesthetic induction agents such as propofol and alfaxalone are recommended for dogs with BOAS for a smooth induction of anesthesia, rapid endotracheal (ET) intubation, and airway control. ${ }^{60}$ These drugs are relatively short acting, thus allowing a quick recovery. However, anesthetic induction can be associated with respiratory depression and apnea, which can lead to oxygen desaturation and cyanosis. ${ }^{63}$ This can be a particular problem as brachycephalic dogs can be challenging to intubate due to their conformation. The excessive oropharyngeal tissue, elongated soft palate, oversized tongue, and laryngeal collapse, when present, all obscure or narrow the airway to make intubation difficult. It is recommended that brachycephalic dogs are pre-oxygenated prior to anesthesia. This will delay desaturation in dogs that are apneic following induction or if intubation is difficult and delayed. ${ }^{64}$ For intubation, a range of sizes of ET tubes should be available, as some brachycephalic dogs require a smaller tube than expected (particularly those with tracheal hypoplasia). In some instances, a stylet can be placed in the ET tube to increase the rigidity and thus make intubation easier. The use of a laryngoscope is essential to facilitate intubation. A second laryngoscope or tongue depressor is very useful to push soft tissues out of the way to allow a good view of the larynx. Once the ET tube is placed, the cuff should be inflated to protect the airway from aspiration as these dogs may be at risk of regurgitation. Once anesthetized, capnography can be very useful to make sure there is no obstruction of the ET tube, which can occur due to excessive secretions often seen in dogs with BOAS.

Many texts recommend the use of perioperative glucocorticoids (dexamethasone) to reduce postoperative swelling and edema. Although many surgeons use glucocorticoids routinely, specific evidence for their efficacy in this context is lacking.

For all BOAS surgeries, the dog should be positioned in sternal recumbency, with the maxilla suspended, the mandible secured to the operating table, and the ET tube secured to ensure free access to the soft palate. The use of a mouth gag for intraoral surgery is recommended.

\section{Soft palate}

Soft palate resection (staphylectomy) is commonly performed in order to shorten the overlong soft palate. The soft palate is often edematous, inflamed, and hypertrophied in affected dogs ${ }^{65}$ overlying the epiglottis by more than a few millimeters. Soft palate resection has been documented as early as the 1920s, with traditional resection techniques involving sharp excision of a portion of the overly long soft palate, and then opposing the oral and nasal mucosal edges of the new free edge of the soft palate with sutures..$^{17,66-71}$ A wide variety of different techniques are described for staphylectomy, including the use of an electroscalpel, ${ }^{23,51}$ radiofrequency,${ }^{72}$ laser ${ }^{73}$ and bipolar sealing device. ${ }^{74}$ All are associated with a good clinical outcome, although the use of a laser has been shown to be significantly faster than sharp dissection. ${ }^{74}$ Folded flap palatoplasty is a recently described alternative procedure, where the bulk of the palatine muscles are reduced in addition to shortening the palate. ${ }^{75}$ This technique is designed to address not only the length but also the thickness or "bulk" of the palate. While the technique is well described, there is limited objective evidence comparing this with more traditional techniques. The timing of staphylectomy is considered important, with dogs less than 2 years of age at the time of soft palate resection more likely to be improved (91\%) compared to those 2 years or older (68\%) (Figure 7). ${ }^{76}$ 


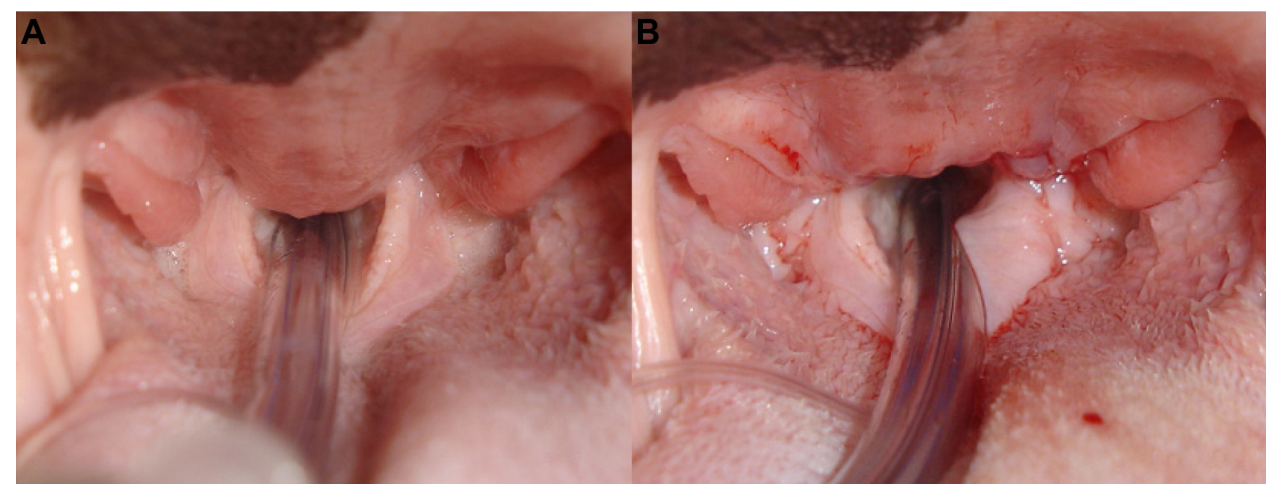

Figure 7 Elongated soft palate.

Notes: (A) Pre- and (B) postsurgical resection.

\section{Stenotic nares}

Rhinoplasty can surgically enlarge stenotic nares. To reduce the chance of secondary airway changes occurring, recommendations for this procedure have been suggested for affected pups as young as 3-4 months old. ${ }^{21}$ If both nasal and soft palate surgeries are carried out, then clinical signs are more likely to be improved than soft palate surgery alone ${ }^{68}$ The original correction technique was described by Trader, ${ }^{77}$ where a wedge of the alar fold (both skin and cartilage) is resected without the use of sutures. ${ }^{78}$ Although reported as effective, the cosmetic result of this process is not seen as esthetically pleasing, and has fallen out of favor. ${ }^{78}$ Modified techniques have also been utilized, including the use of sutures ${ }^{6,79,80}$ and using alternative equipment such as an electroscalpel. ${ }^{23}$ Several wedge resection (alaplasty) techniques are described that improve airflow, while maintaining a cosmetic appearance. These surgeries, such as the vertical, ${ }^{81-83}$ horizontal, ${ }^{32}$ and lateral ${ }^{48}$ techniques, excise different parts of the ala nasi, with the aim of reducing the thickness of the alae and increasing the size of the nostril. Good success rates are reported; for example, following wedge resection with suturing, $25 \%$ of dogs were considered to be "breathing normally" postoperatively, with a further 53.6\% deemed to have made a "considerable improvement" ${ }^{80}$ More recently, a surgical technique "alapexy" has been developed, for dogs where wedge resection techniques fail or having very flaccid alar cartilages. This technique causes the ala nasi to be permanently fixed in an abducted, opened position. ${ }^{84} \mathrm{In}$ four out of five cases undergoing this surgery, the outcome was considered successful, with significant improvements in all dogs 24 months postoperatively. ${ }^{84}$ The most recently described technique is punch resection alaplasty, ${ }^{85}$ where a dermatological punch tool removes a circular plug of tissue from the ala nasi. The open tissue edges are then apposed and sutured, causing the ala nasi to be abducted. Postsurgically, the outcomes of ten out of 12 dogs were considered "excellent" and two out of 12 "good" (Figure 8). ${ }^{85}$

\section{Laryngeal saccule resection}

In dogs with grade I laryngeal collapse, the laryngeal saccules are everted, causing additional airway obstruction. This can be treated via surgical resection of the everted saccules (sacculectomy). This procedure, unless occurring in isolation, ${ }^{86}$

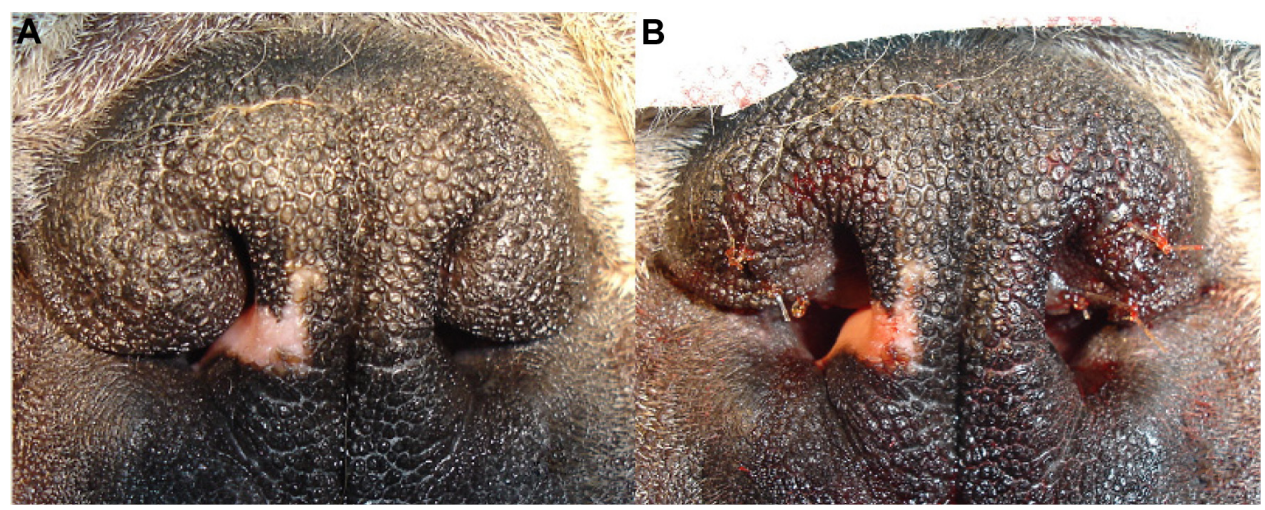

Figure 8 Stenotic nares.

Notes: (A) Pre- and (B) postsurgical correction. 
is usually corrected in addition to soft palate resection and rhinoplasty. ${ }^{51}$ This relatively straightforward procedure, first described by Leonard, ${ }^{87}$ involves resection of the everted saccules with long-handled laryngeal cup forceps, and then removal of any remaining tags of tissue with scissors. ${ }^{88}$ Out of 23 dogs that underwent this procedure (all of which underwent staphylectomy and eight had concurrent rhinoplasty), $78 \%$ showed improvement or were "breathing normally". ${ }^{88}$ Other procedures have been described in the literature, using equipment such as electroscalpels and scissors (eg, Metzenbaum scissors ${ }^{53}$ ), and alternative methods, including twisting each saccule prior to resection to reduce hemorrhage. ${ }^{89}$

The prognosis for early-stage laryngeal collapse is mixed, with reports of only $40 \%$ of dogs undergoing sacculectomy showing a marked improvement. ${ }^{25}$ Over $14 \%$ of dogs undergoing sacculectomy died within 36 hours of this procedure, with six deaths due to aspiration pneumonia. ${ }^{25}$ The authors suggested that secondary changes may have a negative influence on the overall results of surgery; ${ }^{25}$ however, the majority of deaths were in the Bulldogs, and thus, the prognosis may have been influenced by breed and other factors rather than solely saccule eversion.

\section{Advanced laryngeal collapse}

Laryngeal collapse is a secondary problem, which can be very challenging to manage. Dogs with stage II collapse may achieve a moderate improvement through surgical correction of primary abnormalities, resection of everted saccules, and husbandry changes, for example, weight loss and exercise restriction alone. Arytenoidectomy can also be used as corrective surgery to open up the rima glottidis, ${ }^{29}$ with over three quarters $(76.4 \%)$ of patients with stage II collapse having a favorable surgical outcome. ${ }^{27}$ However, the likelihood of improvement for dogs with end-stage laryngeal collapse is markedly reduced. Treatment options are limited with salvage procedures such as partial laryngectomy, permanent tracheostomy, and laryngeal tie back reported. The prognosis for severely affected animals is guarded..$^{90,91}$ For example, $50 \%$ of dogs undergoing partial laryngeal resection died postoperatively, with aspiration pneumonia as a common complication. ${ }^{76}$ An alternative is permanent tracheostomy to bypass the upper airway; however, this requires a high level of attention and maintenance from owners. Permanent tracheostomy may be particularly of high risk in brachycephalic breeds due to their conformation, with short, thick necks in breeds such as the Bulldog, French Bulldog, and Pug and redundant skin folds in the region, resulting in a small stoma. This may be compounded by tracheal hypoplasia when present. As such, euthanasia may be considered by owners for severely affected dogs. Silicone tracheal stoma stents have recently been trialed for the maintenance of a temporary tracheostomy stoma in dogs with upper airway obstruction. The stents were used long term in five dogs before conversion to permanent tracheostomy 5 days to 8 months post-placement; however, such long-term use was not recommended by the authors due to granulation tissue forming in $60 \%$ of the dogs causing dislodgement of the stent. ${ }^{92}$

\section{Further procedures}

In addition to laryngeal collapse, eversion of the tonsils is common in dogs with BOAS and can be treated by tonsillectomy. ${ }^{93}$ This procedure is not commonly performed, and the benefit is unclear, ${ }^{26,28}$ and in some dogs, the everted tonsils return to the tonsil crypts if the primary abnormalities are treated. ${ }^{50}$ One of the drawbacks of traditional surgical management of BOAS is that they do not address the intranasal disease, despite the fact that significant obstruction may be present. A novel procedure has been described to treat aberrant nasal turbinates - laser-assisted turbinectomy. However, this is a very specialized procedure, and regrowth of the turbinates may occur. ${ }^{42,44}$

\section{Postoperative care}

Postoperative care is extremely important following airway surgery for BOAS, and many dogs will require strict observation and intensive nursing. Due to airway compromise, these dogs are most at risk during recovery from anesthetic. In addition, respiratory compromise may be exacerbated by further inflammation and edema as a result of the surgery. Extubation of the ET tube should be delayed for as long as possible and should not occur while the dogs are still sedated to reduce the risk of airway obstruction. ${ }^{60}$ Typically, it is postponed until they can swallow and protect their own airway. ${ }^{60}$ Dogs should be positioned in sternal recumbency with the head elevated to reduce risk of regurgitation and aspiration of fluid. They should be closely monitored following extubation with regular assessment of their respiratory rate and pattern. Any signs of respiratory compromise should be addressed immediately. An intravenous catheter should be maintained postoperatively in case there is a need for sedation or anesthesia. In addition, equipment for re-intubation including induction agent should be available. If an animal does experience respiratory obstruction following extubation, then it should be anesthetized and re-intubated.

Supplemental oxygen is beneficial in the recovery period. Due to the upper respiratory obstruction in these dogs, 
oxygen administration directly into the trachea has significant advantages. ${ }^{94}$ It has been shown that placement of a catheter percutaneously into the trachea can be used to deliver oxygen and is effective at increasing the fraction of inspired oxygen and increasing arterial oxygen partial pressure. ${ }^{94}$ This is a useful technique in an emergency. An alternative is the use of a nasotracheal tube. ${ }^{95}$ This is a simple technique that involves the placement of a naso-esophageal feeding tube through the nose and into the trachea under general anesthesia. This can be placed following surgical correction of BOAS and then used to provide supplemental oxygen in the postoperative period. Nasotracheal tubes have been shown to decrease postoperative respiratory distress but do not seem to affect the incidence of complications or mortality. ${ }^{95}$ Withholding food and water for 12-24 hours post-surgery is suggested by some surgeons but is not widely adopted. ${ }^{90}$

For dogs with severe BOAS or those with postoperative complications related to airway swelling, a temporary tracheostomy tube can be placed. However, the proportion of dogs requiring temporary tracheostomy is relatively low at $1.5 \%-6.8 \% .^{18,27,28}$ Tracheostomy tubes in dogs are known to have a high complication rate, with Bulldogs significantly more likely to have an unsuccessful tube outcome than other breeds. ${ }^{96}$

Postoperative complications include dyspnea, vomiting, and/or regurgitation, wound dehiscence, and coughing and are reported in $6.5 \%-26.2 \%$ of dogs. ${ }^{18,27,28,93}$ Aspiration pneumonia is a risk following surgery, although this has not been specifically reported in more recent publications. Overall mortality rate for surgical correction of BOAS ranges from $0 \%$ to $3.3 \% .^{18,27,28}$

\section{Postoperative outcomes and QoL}

The success of surgical interventions is variable. However, a lack of consistency between scoring systems makes direct comparison challenging (Table 1). This is compounded by variable population demographics between studies (eg, breed distribution and dog age), the number of abnormalities present, the severity of the abnormalities, and different procedures used by different surgeons. Many studies are flawed by the fact that they are investigating one aspect of the disease when it is not possible to standardize the other components and their treatment. Hendricks ${ }^{35}$ noted that owners should not expect dogs to be able to sustain high levels of activity postsurgery, indicating that although clinical signs may improve, dogs that underwent BOAS surgical correction may still be restricted in their activities compared with unaffected animals. Indeed, nearly half of the dogs in one study from North America ${ }^{18}$ and nearly one-third of dogs from another study from Italy ${ }^{33}$ showed an improvement in clinical signs but with residual limits on physical activity.

Breathlessness (dyspnea) is an aversive experience, with "air hunger" being one of three types of unpleasant sensation associated with breathlessness, along with increased work

Table I Degree of improvement observed postsurgery in four studies of brachycephalic obstructive airway syndrome, with categories as defined by the authors of the studies divided into four outcomes

\begin{tabular}{|c|c|c|c|c|}
\hline $\begin{array}{l}\text { Study, number of } \\
\text { patients, and location }\end{array}$ & Worsened & Neutral & Slight improvement & Marked improvement \\
\hline De Lorenzi et al, ${ }^{33}$ & \multicolumn{2}{|l|}{ Poor } & Moderate & Adequate \\
\hline $\mathrm{n}=40$, Italy & \multicolumn{2}{|c|}{$\begin{array}{l}\text { Clinical situation worsened or did not } \\
\text { improve after surgery }\end{array}$} & $\begin{array}{l}\text { Good improvement } \\
\text { of the clinical situation } \\
\text { with some limitations } \\
30.6 \%\end{array}$ & $\begin{array}{l}\text { Excellent improvement in } \\
\text { both the clinical situation and } \\
\text { physical activity after surgery } \\
58.3 \%\end{array}$ \\
\hline \multirow{3}{*}{$\begin{array}{l}\text { Riecks et al, }{ }^{18} \\
n=62, \text { USA }\end{array}$} & Poor & Fair & Good & Excellent \\
\hline & $\begin{array}{l}\text { Severity of clinical signs } \\
\text { increased after surgery }\end{array}$ & No improvement & $\begin{array}{l}\text { Improvement in clinical } \\
\text { signs with some limits } \\
\text { on physical activity }\end{array}$ & $\begin{array}{l}\text { Marked improvement } \\
\text { in clinical signs with no } \\
\text { restriction on physical activity }\end{array}$ \\
\hline & $2.94 \%$ & $2.94 \%$ & $47.1 \%$ & $47.1 \%$ \\
\hline \multicolumn{2}{|l|}{ Torrez and Hunt, ${ }^{27}$} & “l” & "2” & “3” \\
\hline \multirow[t]{2}{*}{$\mathrm{n}=73$, Australia } & & No improvement & Some improvement & Marked improvement \\
\hline & & $10.9 \%$ & $32.6 \%$ & $56.5 \%$ \\
\hline \multirow{3}{*}{$\begin{array}{l}\text { Lorinson et } a{ }^{25} \\
n=118, \text { USA and UK }\end{array}$} & & Poor & Good & Excellent \\
\hline & & $\begin{array}{l}\text { Little or no improvement } \\
\text { was noted following } \\
\text { surgery }\end{array}$ & $\begin{array}{l}\text { Some remaining signs } \\
\text { were seen but were of } \\
\text { a minor nature }\end{array}$ & All signs were ameliorated \\
\hline & & $41.1 \%$ & $14.3 \%$ & $30.4 \%$ \\
\hline
\end{tabular}

Note: Disease severity and surgical procedures performed were variable. 
load and chest tightness. Air hunger is considered to be the most unpleasant sensation of breathlessness in humans, with the greatest potential to compromise animal welfare.$^{97}$ As air hunger is a subjective experience, it can only be self-reported in humans, and thus, whether dogs also experience this unpleasant sensation while in respiratory distress is unknown. In the absence of evidence, to protect animal welfare, we may work on the assumption that when dogs experience respiratory distress, they may experience air hunger, and thus work to prevent it. Air hunger arises primarily from a mismatch between automatic motor command and the tidal volume, ${ }^{98}$ and thus, surgeries must aim to increase the tidal volume of affected dogs to reduce air hunger and associated clinical signs. This can be objectively assessed using barometric whole-body plethysmography (BWBP). ${ }^{99}$ However, this method requires further development and validation in brachycephalic dogs pre- and postsurgery before it can be widely adopted in a clinical setting.

\section{Prospects for the medical management of health issues in brachycephalic dogs Medical management}

The use of medication in BOAS treatment is mainly for the management of acute respiratory crises, where sedation, cooling, and supplemental oxygen may be needed. Both glucocorticoids and diuretics have been suggested for the palliation of advanced disease. ${ }^{90}$ However, there is no evidence for the use of these medications at present, with studies needed to confirm their efficacy.

It should be acknowledged that the impact of BOAS is not limited to the respiratory system, with secondary esophageal and gastric problems common in dogs with BOAS. Moderate-to-severe gastrointestinal (GI) signs, such as hypersalivation, gagging, and retching, have been reported in a significant proportion of dogs with BOAS. ${ }^{26,28}$ It is presumed that negative airway pressure during respiration leads to secondary hiatal hernia, gastroesophageal reflux, and esophagitis. In a long-term study of brachycephalic dogs undergoing combined upper airway surgery and GI tract medical treatment, GI signs were treated using a combination of antacid and prokinetic drugs, dependent upon the results of endoscopic and histological examination. ${ }^{28}$ For inflammatory GI disease observed endoscopically, omeprazole and cisapride were administered postsurgically, and if distal esophagitis was noted, an antacid such as magnesium hydroxide was prescribed after meals for 15 days.
Following histological results, dogs with severe gastritis and/or duodenitis with parietal fibrosis were prescribed omeprazole as an inhibitor of hydrogen ion secretion, cisapride as a prokinetic, sucralfate as a surface protector, and prednisolone for 3 months. For less severe cases, the same treatment was given for 2 months, without the use of prednisolone. After a minimum of 6-month follow-up, $72.1 \%$ of owners considered their dogs' digestive status as "excellent", with $75 \%$ of cases treated for GI signs no longer requiring medical treatment or a special diet. ${ }^{28}$

Due to the potential risks associated with surgical management of BOAS, nonsurgical management should be considered before any surgical intervention, particularly in mild cases, as some patients may improve with exercise restriction and weight loss. Lifestyle changes may reduce the severity of clinical signs; for example, owners should be advised to avoid exposing dogs to activities and environments that markedly increase oxygen demand, in particular excessive exercise, hot weather, and stressful situations. Instead, walks should be short, only at cool times of the day, and harnesses should be used so as not to apply pressure to the upper airway. Obesity is prevalent in the general canine population, affecting an estimated $20 \%-40 \%$ of dogs. ${ }^{100}$ Many dogs with BOAS are overweight or obese. This is problematic as a high body condition score is a risk factor for BOAS ${ }^{16}$ and is significantly associated with an increased severity of clinical signs. ${ }^{26}$ In addition, BWBP studies have demonstrated that obese dogs had a significantly decreased tidal volume per kilogram and significantly increased respiratory rate compared with nonobese dogs. ${ }^{101}$ Owners should be educated to keep their dogs lean to avoid exacerbating clinical signs (Figure 9).

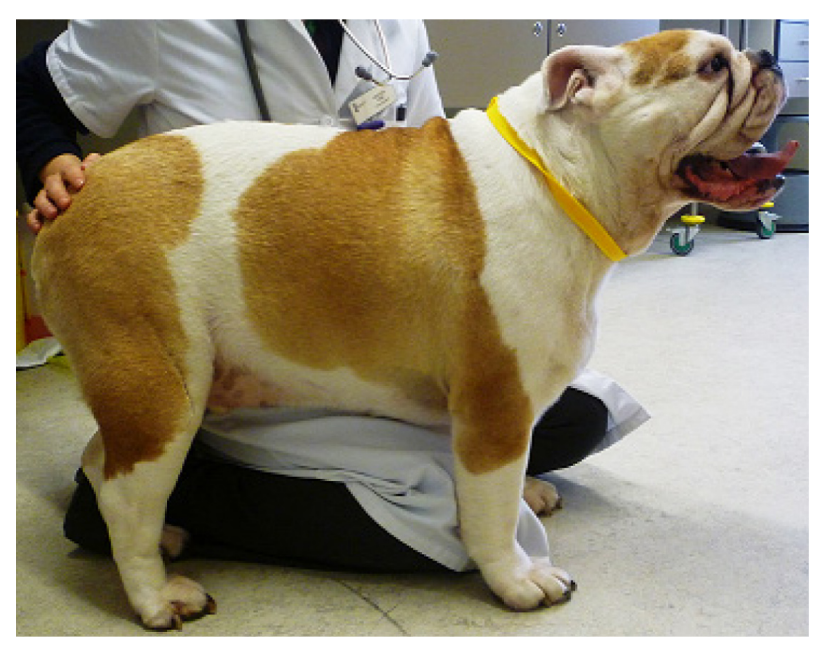

Figure 9 Obese Bulldog diagnosed with brachycephalic obstructive airway syndrome. 


\section{BOAS education}

BOAS education is not limited to encouraging owners to keep their dogs lean. Education of owners, breeders, and the puppy-buying public regarding the perception of BOAS as a serious respiratory problem is needed for several reasons, including the following:

1) To avoid the undertreatment of dogs affected by BOAS to improve individual brachycephalic dog welfare;

2) To avoid the use of BOAS-affected dogs in breeding programs to avoid the perpetuation of this disorder;

3) To ensure that puppy buyers are aware of this condition if choosing to buy a brachycephalic dog.

A recent study demonstrated that over half (58\%) of owners recognized the clinical signs of BOAS in their dogs but dismissed these respiratory difficulties as "normal for the breed". This is worrying as it suggests that many dogs with BOAS may not receive the veterinary attention they need to improve their QoL. Reasons for this perception of "normality" might include the high prevalence of BOAS in brachycephalic breeds, its relatively early onset, and chronicity of clinical signs. ${ }^{4}$ Avoiding undertreatment of this disorder should be the priority of all veterinary surgeons who come into contact with brachycephalic dogs, ensuring that their clients are vigilant of respiratory problems in their dog and seek advice before significant deterioration has occurred. Highlighting detectable respiratory abnormalities to owners of brachycephalic dogs presented for other complaints, for example, audible stertor or excessive panting, should be commonplace in veterinary practice, to ensure that owners take these signs seriously. Although no official health-testing schemes (such as those ran by the British Veterinary Association and the Kennel Club) are available for BOAS in the UK at present, if clients intend to breed from their brachycephalic dog, vets should provide an assessment of their dog's physical health before this can be recommended. Avoiding the use of dogs with obvious clinical signs of BOAS, along with avoiding those with extreme craniofacial morphologies (ie, muzzle length) and severe stenosis of the nares, ${ }^{16}$ may go some way in reducing BOAS risk in resultant litters. Finally, vets can play a part in changing the market forces associated with puppy buying, by educating potential owners as to what they should avoid in a brachycephalic dog, to create a demand for healthy dogs, free of BOAS. Although this may be challenging at the individual dog level, as many owners do not seek veterinary advice until they have already bought a puppy, veterinarians and other animal professions working in educational roles, such as in animal welfare charities, may have access to a wider audience to disseminate this message.
Despite the major welfare impact of BOAS upon affected dogs, many veterinarians are thought to be desensitized to BOAS, accepting it as normal for these breeds. ${ }^{102,103}$ These attitudes must be tackled, with education beginning in vet schools and continuing through ongoing continuing professional development, instilling a serious attitude toward conformational disorders.

\section{Conclusion}

BOAS is extremely prevalent in brachycephalic dog breeds, and this coupled with their increasing popularity creates a major welfare issue. The owner and public awareness of this condition and effective treatments to reduce its effects on QoL are needed more than ever. Ensuring that owners of affected dogs are aware of the poor prognosis and lack of effective treatment options associated with secondary changes is of importance so that dogs are presented in a timely manner, at a stage where surgical options with a good prognosis are still available. Although owners may be hesitant to opt for surgical treatment due to the inherent anesthetic risk of these dogs, these risks must be weighed against the chronic negative effects that BOAS may have upon the affected dog's QoL if left untreated. Although surgery has proven to be effective in alleviating a degree of airway obstruction, affected dogs cannot be made truly "normal" due to their inherent conformation, and thus, emphasis on better breeding of brachycephalics with a focus on health is also required. ${ }^{104}$ Recent studies have demonstrated the major role of conformation in the promotion and perpetuation of BOAS, confirming its status as a "man-made" disorder. ${ }^{16}$ Strong leadership from dog-breeding organizations worldwide is required, to discourage extremes of morphology that lead to this disorder, which may include amendments to the breed standards of affected breeds and health schemes to ensure that only healthy dogs are used for breeding. The veterinary community must provide guidance to breeders and owners, encouraging the prevention rather than palliation of this disorder, ensuring that they do not perpetuate BOAS through desensitized attitudes.

\section{Disclosure}

The authors declare no conflicts of interest related to this paper.

\section{References}

1. The Kennel Club. Breed Registration Statistics. 2014; Available from http://www.thekennelclub.org.uk/registration/breed-registrationstatistics. Accessed September 1, 2014.

2. Pollinger JP, Bustamante CD, Fledel-Alon A, Schmutz S, Gray MM, Wayne RK. Selective sweep mapping of genes with large phenotypic effects. Genome Res. 2005;15(12):1809-1819. 
3. Stockard CR. The Genetic and Endocrinic Basis for Differences in form and Behavior: As Elucidated by Studies of Contrasted Pure-Line Dog Breeds and Their Hybrids. Vol 19. Philadelphia: The Wistar Institute of Anatomy and Biology; 1941.

4. Packer RMA, Hendricks A, Burn CC. Do dog owners perceive the clinical signs related to conformational inherited disorders as 'normal' for the breed? A potential constraint to improving canine welfare. Anim Welf. 2012;21(Suppl 1):81-93.

5. Evans HE. Miller's Anatomy of the Dog. Philadelphia: Saunders; 1993.

6. Brehm H, Loeffler K, Komeyli H. The shapes of the canine skull. Anat Histol Embryol. 1985;14:324-331.

7. Regodon S, Vivo J, Franco A. Craniofacial angle in dolicho-, meso- and brachycephalic dogs: radiological determination and application. Ann Anat. 1993;175:361-363.

8. American Kennel Club. The Complete Dog Book. 20 ed. New York, NY: Ballatine Books; 2006.

9. Ellis JL, Thomason J, Kebreab E, Zubair K, France J. Cranial dimensions and forces of biting in the domestic dog. J Anat. 2009;214(3): $362-373$.

10. Noller C, Hueber JP, Aupperle H, et al. New Aspects of Brachycephalia in Dogs and Cats. Basics: Insights into Embryology, Anatomy and Pathophysiology. Universitat Leipzig, Leipzig, Germany; 2008.

11. Lorenz K. From Studies in Animal and Human Behavior. Vol II. London: Methuen and Co, Ltd; 1971.

12. The Kennel Club. Pug Breed Standard. 2012; Available from: http://www.thekennelclub.org.uk/services/public/breed/standard. aspx ?id=6164. Accessed November 1, 2012.

13. The Kennel Club. Bulldog Breed Standard. 2010; Available from: http://www.thekennelclub.org.uk/services/public/breed/standard. aspx?id=4084. Accessed November 1, 2012.

14. The Kennel Club. Pekingese Breed Standard. 2008; Available from: http://www.thekennelclub.org.uk/services/public/breed/standard. aspx?id=6162. Accessed November 1, 2012.

15. Oechtering GU. Brachycephalic syndrome: new information on an old congenital disease. Vet Focus. 2010;20(2):2-9.

16. Packer RMA, Hendricks A, Tivers MS, Burn CC. Short muzzle; short of breath? The effect of conformation on the risk of brachycephalic obstructive airway syndrome (BOAS) in domestic dogs. Paper presented at: UFAW International Animal Welfare Science Symposium; 4-5th July, 2013; Universitat Autònoma de Barcelona, Barcelona, Spain.

17. Singleton WB. Partial velum palatiectomy for relief of dyspnea in brachycephalic breeds. J Small Anim Pract. 1962;3:215-216.

18. Riecks TW, Birchard SJ, Stephens JA. Surgical correction of brachycephalic syndrome in dogs: 62 cases (1991-2004). J Am Vet Med Assoc. 2007;230(9):1324-1328.

19. Hendricks JC, editor. Recognition and treatment of congenital respiratory tract defects in brachycephalics. In: Bonagura JD, editor. Kirk's Veterinary Therapy XII. Philadelphia: WB Saunders; 1995:892.

20. Dupre GP. Brachycephalic syndrome: new knowledge, new treatments. In: 33rd World Small Animal Veterinary Association Congress; August 20-24, 2008, 2008; Dublin, Ireland.

21. Aron DN, Crowe DT. Upper airway-obstruction - general-principles and selected conditions in the dog and cat. Vet Clin North Am Small Anim Pract. 1985;15(5):891-917.

22. Hendricks JC, Kline LR, Kovalski RJ, O’Brien JA, Morrison AR, Pack AI. The English bulldog: a natural model of sleep-disordered breathing. J Appl Physiol. 1987;63(4):1344-1350.

23. Knecht CD. Upper airway obstruction in brachycephalic dogs. Comp Cont Educ Pract Vet. 1979;1:25-30.

24. Harvey CE. Inherited and congenital airway conditions. J Small Anim Pract. 1989;30(3):184-187.

25. Lorinson D, Bright RM, White R. Brachycephalic airway obstruction syndrome - a review of 118 cases. Canine Pract. 1997;22(5-6): $18-21$.
26. Poncet CM, Dupre GP, Freiche VG, Estrada MM, Poubanne YA, Bouvy BM. Prevalence of gastrointestinal tract lesions in 73 brachycephalic dogs with upper respiratory syndrome. J Small Anim Pract. 2005;46(6):273-279.

27. Torrez CV, Hunt GB. Results of surgical correction of abnormalities associated with brachycephalic airway obstruction syndrome in dogs in Australia. J Small Anim Pract. 2006;47(3):150-154.

28. Poncet CM, Dupre GP, Freiche VG, Bouvy BM. Long-term results of upper respiratory syndrome surgery and gastrointestinal tract medical treatment in 51 brachycephalic dogs. J Small Anim Pract. 2006;47(3): $137-142$.

29. Wykes PM. Brachycephalic airway obstructive syndrome. Probl Vet Med. 1991;3(2):188-197.

30. Holt DE. Upper airway obstruction, stertor, and stridor. In: King LG, editor. Textbook of Respiratory Disease in Dogs and Cats. St Louis: Elsevier; 2004:35-42.

31. Ohnishi T, Ogura JH. Partitioning of pulmonary resistance in the dog. Laryngoscope. 1969;79:1847-1878.

32. Leonard HC. Surgical relief for stenotic nares in a dog. J Am Vet Med Assoc. 1956;128:530.

33. De Lorenzi D, Bertoncello D, Drigo M. Bronchial abnormalities found in a consecutive series of 40 brachycephalic dogs. J Am Vet Med Assoc. 2009;235(7):835-840.

34. Oechtering GU. Brachycephalics - trapped in man-made misery? Paper presented at: XXIV Symposium on Current Diseases of Small Animals; 14-16th April 2011, 2011; Portoroz, Slovenia.

35. Hendricks JC. Brachycephalic airway syndrome. Vet Clin North Am Small Anim Pract. 1992;22(5):1145-1153.

36. Goldberg M, Langman V, Taylor C. Panting in dogs: paths of air flow in response to heat and exercise. Respir Physiol. 1981;43(3):327-338.

37. Schmidt-Nielsen K, Bretz W, Taylor C. Panting in dogs: unidirectional air flow over evaporative surfaces. Science. 1970;169:1102-1104.

38. Baile E, Guillemi S, Paré P. Tracheobronchial and upper airway blood flow in dogs during thermally induced panting. J Appl Physiol. 1987; 63(6):2240-2246.

39. Blatt C, Taylor C, Habal M. Thermal panting in dogs: the lateral nasal gland, a source of water for evaporative cooling. Science. 1972;177:804-805.

40. Oechtering GU, Schluter C, Lippert JP. Brachycephaly in dog and cat: a 'human induced' obstruction of the upper airways. Pneumologie. 2010;64:450-452.

41. Negus V. The Comparative Anatomy and Physiology of the Nose and Paranasal Sinuses. London: E and S Livingstone Ltd; 1958.

42. Ginn JA, Kumar MSA, McKiernan BC, Powers BE. Nasopharyngeal turbinates in brachycephalic dogs and cats. J Am Anim Hosp Assoc. 2008;44(5):243-249.

43. Hueber J, Smith H, Reinhold P. Brachycephalic airway syndrome: effects of partial turbinectomy on intranasal airway resistance. Paper presented at: The 25th Symposium of the Veterinary Comparative Respiratory Society 2009; Lafayette, Indiana, USA.

44. Oechtering G, Hueber J, Oechtering T. Laser-assisted turbinectomy (LATE) - treating brachycephalic airway distress at its intranasal origin. Vet Surg. 2007;36:18.

45. Walter A, Seeger J, Oechtering G. Dolichocephalic versus brachycephalic conchae nasales - a microscopic anatomical analysis in dogs. Paper presented at: XXVII Congress of the Association of Veterinary Anatomists 2008; Budapest, Hungary.

46. Lippert JP, Reinhold P, Smith HJ, et al. Geometry and function of the canine nose: how does the function change when the form is changed? Pneumologie. 2010;64(7):452-453.

47. Hueber J. Impulse oscillometric examination of intranasal airway resistance before and after laser-assisted turbinectomy for treatment of brachycephalic airway syndrome in the dog [Doctoral thesis], University of Leipzig; 2008.

48. Monnet E, editor. Brachycephalic airway syndrome. In: Slatter D, editor. Textbook of Small Animal Surgery. Philadelphia: WB Saunders; 2003:808-813. 
49. Orsher RJ, editor. Brachycephalic airway disease. In: Bojrab J, editor. Disease Mechanisms in Small Animal Surgery. 2 ed. Philadelphia: Lea and Febiger; 1993:369.

50. Koch DA, Arnold S, Hubler M, Montavon PM. Brachycephalic syndrome in dogs. Comp Cont Educ Pract Vet. 2003;25(1):48.

51. Leonard HC. Collapse of the larynx and adjacent structures in the dog. J Am Vet Med Assoc. 1960;137:360-363.

52. Cook WR. Observations on the upper respiratory tract of the dog and cat. J Small Anim Pract. 1964;5:309-329.

53. Pink JJ, Doyle RS, Hughes JML, Tobin E, Bellenger C. Laryngeal collapse in seven brachycephalic puppies. J Small Anim Pract. 2006;47(3):131-135.

54. Harvey CE, Fink EA. Tracheal diameter - analysis of radiographic measurements in brachycephalic and non-brachycephalic dogs. $J \mathrm{Am}$ Anim Hosp Assoc. 1982;18(4):570-576.

55. Manens J, Ricci R, Damoiseaux C, et al. Effect of body weight loss on cardiopulmonary function assessed by 6-minute walk test and arterial blood gas analysis in obese dogs. J Vet Intern Med. 2014;28 371-378.

56. Brown D, Gregory S. Brachycephalic Airway Disease. BSAVA; Cheltenham, UK 2005.

57. Hedlund CS. Brachycephalic Syndrome. 4 ed. Baltimore: The Williams \& Wilkins Co; 1998.

58. Murgia D, Pivetta M, Bowlt K, Volmer C, Holloway A, Dennis R. Intranasal epidermoid cyst causing upper airway obstruction in three brachycephalic dogs. J Small Anim Pract. 2014;55(8):431-435.

59. Coyne BE, Fingland RB. Hypoplasia of the trachea in dogs -103 cases (1974-1990). J Am Vet Med Assoc. 1992;201(5):768-772.

60. Adshead S. Reducing the risk of anaesthetic complications in patients with brachycephalic obstructive airway syndrome. Vet Nurse. 2014;5(2): 78-87.

61. Clutton R. Respiratory disease. In: Seymour C, Duke-Novakovski T, editors. BSAVA Manual of Canine and Feline Anaesthesia and Analgesia. Gloucester: BSAVA; 2007:200-219.

62. Thomson S. Ophthalmic surgery. In: Seymour C, Duke-Novakovski T, editors. BSAVA Manual of Canine and Feline Anaesthesia and Analgesia. Gloucester: BSAVA; 2007:183-194.

63. Amengual M, Flaherty D, Auckburally A, Bell A, Scott E, Pawson P. An evaluation of anaesthetic induction in healthy dogs using rapid intravenous injection of propofol or alfaxalone. Vet Anaesth Analg. 2013;40(2):115-123.

64. McNally E, Robertson S, Pablo L. Comparison of time to de-saturation between pre-oxygenated and non preoxygenated dogs following sedation with acepromazine maleate and morphine and induction of anaesthesia with propofol. Am J Vet Res. 2009;70(11):1333-1338.

65. Pichetto M, Arrighi S, Roccabianca P, Romussi S. The anatomy of the dog soft palate. II. Histological evaluation of the caudal soft palate in brachyephalic breeds with grade I brachycephalic airway obstruction syndrome. Anat Rec (Hoboken). 2011;294(7):1267-1272.

66. Schlotthauer CF. Congenital defects in the stomach of a dog. J Am Vet Med Assoc. 1929;75:370-371.

67. Farquharson J, Smith DW. Resection of the soft palate in the dog. J Am Vet Med Assoc. 1942;100:427-430.

68. Harvey CE. Soft palate resection in brachycephalic dogs. J Am Anim Hosp Assoc. 1982;18(4):538-544.

69. Wallace LJ. Cleft palate surgery in the dog. Proc Am Anim Hosp Assoc. 1972;39:641-644.

70. Hofmeyr CFB. Surgery of the pharynx. Vet Clin Small Anim Pract. 1972;2:3-16.

71. Baker GJ. Surgery of the canine pharynx and larynx. J Small Anim Pract. 1972;13:505-513.

72. Elkins AD. Soft palate resection in brachycephalic dogs. Vet Forum. 2005;22:43-46.

73. Clark GN, Sinibaldi KR. Use of a carbon dioxide laser for treatment of elongated soft palate in dogs. J Am Vet Med Assoc. 1994;204(11): 1779-1781.
74. Davidson EB, Davis MS, Campbell GA. Evaluation of carbon dioxide laser and conventional incisional techniques for resection of soft palates in brachycephalic dogs. J Am Vet Med Assoc. 2001;219: 776-781.

75. Findji L, Dupre G. Folded flap palatoplasty for treatment of elongated soft palates in 55 dogs. Vet Med Austria. 2008;95:56-63.

76. Harvey C. Upper airway obstruction surgery. II. Soft palate resection in brachycephalic dogs. J Am Anim Hosp Assoc. 1982;18: 538-544.

77. Trader RL. Nose operation. J Am Vet Med Assoc. 1949;114:210-211.

78. Huck JL, Stanley BJ, Hauptman JG. Technique and outcome of nares amputation (trader's technique) in immature shih tzus. J Am Anim Hosp Assoc. 2008;44(2):82-85.

79. O'Brien JA, Harvey CE. Diseases of the Upper Airway Textbook of Veterinary Internal Medicine. Philadelphia: WB Saunders; 1975.

80. Harvey CE. Stenotic nares surgery in brachycephalic dogs. J Am Anim Hosp Assoc. 1982;18(4):535-537.

81. Blakely CL. Repair of nasal cartilage in dogs. North Am Vet. 1951;32:628.

82. Kagan K, editor. Nasal Cavity. In: Bojrab MJ, editor. Current Techniques in Small Animal Surgery. 2 ed. Philadelphia: Lea and Febiger; 1983:371-374.

83. Engen $\mathrm{MH}$, editor. The external nares. In: Bojrab MJ, editor. Current Techniques in Small Animal Surgery. Philadelphia: Lea and Febiger; 1975:375-382.

84. Ellison GW. Alapexy: an alternative technique for repair of stenotic nares in dogs. J Am Anim Hosp Assoc. 2004;40(6):484- 489.

85. Trostel CT, Frankel DJ. Punch resection alaplasty technique in dogs and cats with stenotic nares: 14 cases. J Am Anim Hosp Assoc. 2010;46(1): $5-11$.

86. Rudorf H, Lane JG, Wotton PR. Everted laryngeal saccules: ultrasonographic findings in a young Lakeland terrier. J Small Anim Pract. 1999;40(7):338-339.

87. Leonard HC. Eversion of the lateral ventricles of the larynx in dogs five cases. J Am Vet Med Assoc. 1957;128:83-84.

88. Harvey CE. Everted laryngeal saccule surgery in brachycephalic dogs. J Am Anim Hosp Assoc. 1982;18(4):545-547.

89. Kagan KG. Surgical Management of Laryngeal Obstruction. American College of Veterinary Surgeons Surgical Forum; Chicago, USA 1981.

90. Reiter A, Holt D. Palate. In: Tobias K, Johnston S, editors. Veterinary Surgery Small Animal. Vol II. Missouri: Elsevier Saunders; 2012: 1707-1717.

91. White R. Surgical management of laryngeal collapse associated with brachycephalic airway obstruction syndrome in dogs. J Small Anim Pract. 2012;53:44-50.

92. Trinterud T, Nelissen P, White RAS. Use of silicone tracheal stoma stents for temporary tracheostomy in dogs with upper airway obstruction. $J$ Small Anim Pract. 2014;55(11):551-559.

93. Fasanella F, Shivley J, Wardlaw J, Givaruangsawat S. Brachycephalic airway obstructive syndrome in dogs: 90 cases (1991-2008). J Am Vet Med Assoc. 2010;237:1048-1051.

94. Mann F, Wagner-Mann C, Allert J. Comparison of intra- nasal and intratracheal oxygen administration in healthy awake dogs. Am J Vet Res. 1992;53(5):856-860.

95. Senn D, Sigrist N, Forterre F, Howard J, Spreng D. Retrospective evaluation of postoperative nasotracheal tubes for oxygen supplementation in dogs following surgery for brachycephalic syndrome: 36 cases (2003-2007). J Vet Emerg Crit Care. 2011;21(3):261-267.

96. Nicholson I, Baines S. Complications associated with temporary tracheostomy tubes in 42 dogs (1998 to 2007). J Small Anim Pract. 2012;53(2):108-114.

97. Beausoleil NJ, Mellor DJ. Introducing breathlessness as a significant animal welfare issue. $N$ Z Vet J. 2014;63(1):44-51.

98. Lansing R, Gracely R, Banzett R. The multiple dimensions of dyspnea: review and hypotheses. Respir Physiol Neurobiol. 2009; 167(1):53-60 
99. Bernaerts F, Talavera J, Leemans J, et al. Description of original endoscopic findings and respiratory functional assessment using barometric whole-body plethysmography in dogs suffering from brachycephalic airway obstruction syndrome. Vet J. 2010;183:95-102.

100. Gossellin J, Wren J, Sunderland S. Canine obesity: an overview. JVet Pharmacol Ther. 2007;30:1-10.

101. Manens J, Bolognin M, Bernaerts F, Diez M, Kirschvink N, Clercx C. Effects of obesity on lung function and airway reactivity in healthy dogs. Vet J. 2012;193(1):217-221.
102. Arman K. A new direction for kennel club regulations and breed standards. Can Vet J. 2007;48(9):953-964.

103. Laurence C. Balancing pedigree dog breed standards and animal welfare - is it possible? Vet Rec. 2009;164:481-482.

104. Packer R, Hendricks A, Burn C. Conference Report: Building Better Brachycephalics 2013. North Mymms: Royal Veterinary College; 2014.

\section{Publish your work in this journal}

Veterinary Medicine: Research and Reports is an international, peer-reviewed, open access journal publishing original research, case reports, editorials, reviews and commentaries on all areas of veterinary medicine. The manuscript management system is completely online and includes a very quick and fair peer-review system.
Visit http://www.dovepress.com/testimonials.php to read real quotes from published authors. 